\title{
Quantifying the discrepancy between pre-operative suspicion and intra-operative confirmation of a dural breach in patients with skull fractures post assault
}

\begin{abstract}
Background: Skull fractures are associated with a dural breach and are regarded as Neurosurgical emergencies to prevent potentially devastating infective complications. We aimed to quantify the discrepancy between pre-operative suspicion and intra-operative confirmation of a dural breach in 246 patients admitted over a 2 year period with skull fractures post assault.
\end{abstract}

Materials and Methods: Retrospective data analysis of all patients that were admitted to the neurosurgical wards at our institution with skull fractures post assault from January 2015-December 2016 was performed. Medical charts were analyzed for pre-operative suspicion of a dural breach including the evidence for this suspicion, and operative confirmation of a dural breach. Management included simple suturing and antibiotics or surgery with antibiotic cover.

Results: Of 246 patients treated, 234 (95\%) were male and $12(5 \%)$ were female. The mean age of subjects was $32+/-$ 12 years. In terms of compound skull fracture management $23(9 \%)$ were treated with simple suturing and antibiotics and 223 (91\%) were treated surgically with antibiotics. Considering pre-operative suspicion of a dural breach in all 246 patients in 95 (39\%) of cases a dural breach was suspected and in 151 (61\%) a dural breach was not suspected. In the operative group of 223 patients a dural breach was confirmed in $109(49 \%)$ of cases and excluded in $113(51 \%)$ of cases.

Conclusion: Although a dural breach was suspected in $95(39 \%)$ of all 246 subjects using all available clinical and radiological evidence, in the operative group of 223 subjects a dural breach was confirmed in 113 (49\%) of subjects. Hence the attending surgeon should have a low threshold for surgical exploration of skull fractures secondary to assault for fear of missing approximately $10 \%$ of dural breaches.

\section{Keywords: Predicting dural breach, skull fracture, assault head injury}

\section{Introduction}

A skull fracture that causes a dural breach is considered a neurosurgical emergency to prevent devastating infective consequences that include meningitis, subdural empyema and intracerebral abscess [1]. Recognizing a dural breach and performing a dural repair is such a fundamental neurosurgical consideration several papers support the proposition all compound skull fractures need formal operative exclusion of this being present [1-3].

To date there are no South African papers focused solely on establishing the incidence of dural breach in skull fractures post assault. There is also no South African literature specifically establishing a numerical value to define the discrepancy between the pre-operative clinical or radiological suspicion of a dural breach versus intra-operative confirmation.

We analyzed skull fractures post assault for pre-operative suspicion of a dural breach including the evidence for this suspicion, and operative confirmation of a dural breach, at our neurosurgical unit situated in Gauteng, South Africa, over a 24 month period.

\section{Method and Materials}

This was a retrospective chart review of patients admitted with skull fractures post assault presenting to the Department of Neurosurgery at Dr. George Mukhari Academic Hospital located
Adrian Kelly*, Vanessa Moodley

George Mukhari Academic Hospital, Sefako Makgatho Health Sciences University, Ga-Rankuwa, Pretoria, South Africa

*Author for correspondence:

adriankelly1000@yahoo.co.uk 
in Pretoria, Gauteng, South Africa. The study period was from 01 January 2015-31 December 2016. Ethics approval for the study was granted by the Medical Research Ethics Committee of Sefako Makgatho Health Sciences University; reference number SMUREC/M/38/2017:PG.

In total 246 patients with skull fractures post assault were admitted to our unit during the study period. Our institutional policy is to initially close these wounds in the Emergency department where under local anesthetic cover we perform wound irrigation, a limited debridement, and approximation of the wound edges with simple suturing. Post admission indications for further operative intervention are depressed skull fractures, contaminated compound skull fractures, compound skull fractures with significant scalp damage, presence of brain matter in the wound, cerebrospinal fluid visible or oozing from the wound, associated acute subdural hematomas, extradural hematomas, intracerebral hematomas or intracerebral contusions requiring operative intervention in their own right. These intracranial mass lesions are managed according to the recommendation principles of the brain trauma foundation.org [4]. Frontal sinus injuries that involve the posterior wall [5] as well as septic fractures are further indications for formal operative intervention at our institution. In terms of closed fractures our institutional policy is much more conservative and these are only operated if causing cosmetic concerns or causing a focal deficit by exerting direct pressure on the adjacent cortex.

A craniectomy with/without a dural repair is our operative intervention of choice for open depressed skull fractures. Comminuted bone fragments are rarely replaced due to the risk of sepsis and instead we offer a delayed cranioplasty to our patients between 4 and 6 weeks post operatively. We give special attention to a thorough dural debridement to limit the risk of sepsis and generally find a dural substitute graft necessary to close the defect. For this purpose we use harvested pericranium and insist on a watertight closure.

The data recorded and analyzed in our study included patient demographics, the clinical and/ or radiological pre-operative suspicion of a dural breach including evidence for this suspicion, and operative confirmation of a dural breach.

\section{Results and Discussion}

Skull fractures post assault add considerable burden to the trauma load dealt with by our neurosurgery unit at the Dr. George Mukhari Academic Hospital in Pretoria, South Africa.

In total we admitted 246 patients with skull fractures post assault from 01 January 201531 December 2016. There were 234 (95\%) males and $12(5 \%)$ females. The mean age was $32+/-12$ years. The study results were that almost all of the patients were male similar to the finding in another study by Enicker who, in another South African paper considered that only head injuries incurred by machete injuries, revealing that $93 \%$ of his 185 subjects were male [6]. The findings of Irie, in an Australian study conducted in Queensland that considered traumatic brain injury of which $17 \%$ were due to assault, supports our study finding as well as that by Enickler in that $97 \%$ of the subjects in this study assault group were also male [7].

All $246(100 \%)$ of patients had a skull fracture secondary to assault and in $193(78 \%)$ of subjects this was compound and in $53(22 \%)$ this was a closed fracture. In 153 (62\%) of subjects this fracture was depressed and in 93 (38\%) the fracture was not depressed. The fact that by far the majority of the skull fractures seen were compound as well as the fact that over half of the skull fractures seen were depressed illustrates the need for urgent referral to a neurosurgical center for these injuries as both are indications for Neurosurgical operative intervention.

Considering the pre-operative suspicion of a dural breach in all $246(100 \%)$ subjects this was suspected in $95(39 \%)$ of subjects and was not suspected in 151 (61\%) of subjects. The evidence for this suspicion in these 95 subjects was either on the basis of (1) Clinical examination of the patient or (2) Review of the patient's radiology namely the pre-operative non-contrast CT brain. In terms of the clinical examination a dural breach was suspected in 7 (7\%) of subjects on the basis of visible brain matter in the wound and in $11(12 \%)$ of subjects on the basis of a cerebrospinal fluid leak at the wound site. Hence in total only $18(19 \%)$ of the total $95(100 \%)$ of subjects suspected of having a dural breach had direct clinical evidence on examination. In the remaining 77 (81\%) subjects the suspicion of a dural breach 
was based purely on radiological review. In 43 $(45 \%)$ of these subjects the suspicion was made on the basis of pneumocephalus and in 34 $(36 \%)$ of patients the suspicion was on the basis of in driven bone.

Of the total 246 patient group, 23 (9\%) were treated with simple suturing and antibiotics and $223(93 \%)$ were treated surgically with antibiotics. In this operative group of 223 patients a dural breach was confirmed intraoperatively in 109 (49\%) of patients and was excluded in 113 (51\%) of patients.

Various authors have evaluated the incidence of dural breach in depressed skull fractures and the range reported is from as low as $25 \%$ as reported by Hossain et al [8] to as high as $68 \%$ as reported by Nayak et al [9]. With such a considerable range reported Salia et al looked at predicting dural breach in compound depressed skull fractures based on radiological markers and noted fracture depression (odds ratio 1.3 $\mathrm{p}<0.001$ ), pneumocephalus (odds ratio 2.8 $\mathrm{p}<0.001$ ) and brain contusions (odds ratio 5.5 $\mathrm{p}<0.001)$ to be significantly associated with a dural breach on univariate and multivariate analysis. In this study by Salia et al the incidence of dural breachs in compound depressed skull fractures was $55 \%$ [10]. Our study supports the above study where we confirmed the presence of a dural breach in $49 \%$ of our subjects.

The fact that a dural breach was suspected in $39 \%$ of subjects while intra-operatively $49 \%$ of subjects did in fact have a dural breach, provides the data that in the study, if only clinical and/ or radiological evidence was used, $10 \%$ of dural breaches would in fact have be missed. This study finding provides evidence for the fact that a very low threshold should be entertained for taking compound skull fractures to the operating room to formally exclude a dural breach $[2,3]$. A dural breach carries with it a significant risk of devastating intracranial sepsis that includes the risk of meningitis, subdural empyema and brain abscess and as such should be regarded as an immediate Neurosurgical emergency [1].

Our study showed that approximately three quarters of patients who incurred a skull fracture secondary to assault presented with a compound fracture and in approximately two thirds of these the fracture was depressed. In terms of preventing sepsis two important considerations are, the use of prophylactic antibiotics and, as our study concludes, the role of formal operative intervention to identify and close any dural breach. In our neurosurgical unit we utilize 72 hours of prophylactic antibiotics that target gram positive, gram negative as well as anaerobic bacteria. Intravenous cloxacillin at a dose of $1 \mathrm{~g}$ administered 6 hourly, intravenous ceftriaxone at a dose of $1 \mathrm{~g}$ administered 12 hourly, and intravenous metronidazole at a dose of $500 \mathrm{mg}$ administered 8 hourly, are our prophylactic first line antibiotics of choice covering each of these bacterial groups respectively. In the rare instance that a wound does develop sepsis we will adjust this empiric regimen to directed therapy based on the microbiological culture result. Several papers emphasize the use of prophylactic triple antibiotic therapy in compound skull fractures to prevent the development of sepsis [11]. If sepsis has been formally excluded in the operating room we stop these antibiotics and commence intravenous cefazolin $1 \mathrm{~g}$ administered 8 hourly for 72 hours.

In conclusion we emphasize the importance of formal operative intervention in the context of compound skull fractures to accurately identify and manage any dural breach. As our study showed the rationale for having a low threshold for operative exploration of compound skull fractures is that if only pre-operative suspicion is relied upon, up to $10 \%$ of dural breaches will be missed.

\section{Conflict of Interest}

None of the authors have any conflict of interest with regards to material contained and disseminated in this article.

\section{Funding}

This research did not receive any specific grant from funding agencies in the public, commercial, or not-for-profit sectors. 
References

Win R. Youmans Neurological Surgery 6th Ed. Elsevier Saunders publishers. 3345, 3442-3446 (2011).

Braakman R. Depressed skull fracture: data treatment and follow-up in 225 consecutive cases. J Neurol Neusurg Psy. 35(3), 395-402 (1972).

Demetriades D, Charalambides D, Lakhoo $\mathrm{M}$, et al. Role of prophylactic antibiotics in open and basilar fractures of the skull: a randomized study. Injury. 23(6), 80-377 (1992).

Carney N, Totten AM, O'Reilly C, et al. Guidelines for the management of severe traumatic brain injury, Fourth
Edition. Nursurg. 80(1), 6-15 (2016).

Rodriguez ED, Stanwix MG, Nam AJ, et al. Twenty-six-year experience treating frontal sinus fractures: a novel algorithm based on anatomical fracture pattern and failure of conventional techniques. Plast Reconstr Surg. 122(6), 66-1850 (2008).

Enicker B, Madiba T. Cranial injuries secondary to assault with a machete. Injury. 45(9), 1355-1388 (2014).

Irie F. Epidemiology of traumatic epidural hematoma in young age. $J$ trauma. 71(4), 847-853 (2011).

Hossain MZ, Mondle MS, Hoque MM. Depressed skull fracture: outcome of surgical treatment. J Teach Assoc. 21(2), 6-140 (2008).

Nayak PK, Mahapra AK. Primary reconstruction of depressed skull fracture- The changing scenario. Indian J Neurotrauma. 5(1), 8-35 (2008).

Salia SM, Mersha HB, Aklilu AT, et al. Predicting Dural Breach in compound Depressed Skull Fractures: A Prospective Multicenter Correlational Study. J World Nursurg. 114: e833-e839 (2018).

Wylen EL, Willis BK, Nanda A. Infection rate with replacement of bone fragments in compound depressed skull fractures. J Surg Neurol. 51, 452-457 (1999). 